\title{
Effects of dietary fat modification on oxidative stress and inflammatory markers in the LIPGENE study
}

\author{
Helena Petersson $^{1}$, Ulf Risérus ${ }^{1}{ }^{*}$, Jolene McMonagle ${ }^{2}$, Hanne L. Gulseth ${ }^{3,4}$, Audrey C. Tierney ${ }^{2}$, \\ Sophie Morange ${ }^{5}$, Olfa Helal ${ }^{6,7,8}$, Danielle I. Shaw ${ }^{9}$, Juan A. Ruano ${ }^{10}$, José López-Miranda ${ }^{10}$,
} Beata Kieć-Wilk $^{11}$, Iwona Gołąbek ${ }^{11}$, Ellen E. Blaak ${ }^{12}$, Wim H. M. Saris ${ }^{12}$, Christian A. Drevon ${ }^{4}$, Julie A. Lovegrove ${ }^{9,13}$, Helen M. Roche ${ }^{2}$ and Samar Basu ${ }^{1}$

${ }^{1}$ Department of Public Health and Caring Sciences/Clinical Nutrition and Metabolism, Uppsala University, Uppsala Science Park, 75185 Uppsala, Sweden

${ }^{2}$ Nutrigenomics Research Group, UCD Conway Institute, School of Public Health and Population Science, University College Dublin, Dublin 4, Ireland

${ }^{3}$ Hormone Laboratory, Department of Endocrinology, Oslo University Hospital Aker, University of Oslo, Oslo, Norway

${ }^{4}$ Department of Nutrition, Institute of Basic Medical Sciences, Faculty of Medicine, University of Oslo, Oslo, Norway

${ }^{5}$ Centre d'Investigation Clinique, Aix-Marseille Université, APH-M, Hôpital Conception, Marseille, France

${ }^{6}$ INSERM, U476 Nutrition Humaine et Lipides, Marseille F-13385, France

${ }^{7}$ INRA, UMR1260 Nutriments Lipidiques et Prévention des Maladies Métaboliques, Marseille F-13385, France

${ }^{8}$ Université de la Méditerranée Aix-Marseille 2, Faculté de Médecine, Marseille F-13385, France

${ }^{9}$ Department of Food and Nutritional Sciences, University of Reading, Reading, UK

${ }^{10}$ Lipids and Atherosclerosis Research Unit, Reina Sofía University Hospital, Maimonides Institute for Biomedical Research at Cordoba (IMIBIC), University of Cordoba, Ciber Physiopatology of Obesity and Nutrition (CB06/03), Instituto de Salud Carlos III, Cordoba, Spain

${ }^{11}$ Department of Clinical Biochemistry, Jagiellonian University Medical College, Krakow, Poland

${ }^{12}$ Department of Human Biology, NUTRIM, School for Metabolism, Toxicology and Nutrition, Maastricht University, Maastricht, The Netherlands

${ }^{13}$ Institute for Cardiovascular and Metabolic Research (ICMR), University of Reading, Reading, UK

(Received 9 December 2009 - Revised 13 April 2010 - Accepted 21 April 2010 - First published online 23 June 2010 )

Subjects with the metabolic syndrome (MetS) have enhanced oxidative stress and inflammation. Dietary fat quality has been proposed to be implicated in these conditions. We investigated the impact of four diets distinct in fat quantity and quality on 8-iso-PGF ${ }_{2 \alpha}\left(\right.$ a major $\mathrm{F}_{2}$-isoprostane and oxidative stress indicator), 15-keto-13,14-dihydro- $\mathrm{PGF}_{2 \alpha}$ (15-keto-dihydro-PGF $2 \alpha$, a major $\mathrm{PGF}_{2 \alpha}$ metabolite and marker of cyclooxygenasemediated inflammation) and C-reactive protein (CRP). In a 12-week parallel multicentre dietary intervention study (LIPGENE), 417 volunteers with the MetS were randomly assigned to one of the four diets: two high-fat diets (38\% energy (\%E)) rich in SFA or MUFA and two low-fat high-complex carbohydrate diets $(28 \% \mathrm{E})$ with (LFHCC $n-3)$ or without (LFHCC) $1.24 \mathrm{~g} / \mathrm{d}$ of very long chain $n-3$ fatty acid supplementation Urinary levels of 8 -iso- $\mathrm{PGF}_{2 \alpha}$ and 15-keto-dihydro- $\mathrm{PGF}_{2 \alpha}$ were determined by RIA and adjusted for urinary creatinine levels. Serum concentration of CRP was measured by ELISA. Neither concentrations of 8 -iso- PGF $_{2 \alpha}$ and 15-keto-dihydro-PGF $2 \alpha$ nor those of CRP differed between diet groups at baseline $(P>0.07)$ or at the end of the study $(P>0.44)$. Also, no differences in changes of the markers were observed between the diet groups (8-iso-PGF ${ }_{2 \alpha}, P=0.83 ; 15$-keto-dihydro-PGF ${ }_{2 \alpha}, P=0.45$; and CRP, $P=0.97$ ). In conclusion, a 12 -week dietary fat modification did not affect the investigated markers of oxidative stress and inflammation among subjects with the MetS in the LIPGENE study.

Dietary fat: Oxidative stress: Inflammation: Metabolic syndrome: LIPGENE study

Subjects with type 2 diabetes have enhanced inflammation and oxidative stress $^{(1-3)}$. These conditions may also be involved in the vascular complications of the disease ${ }^{(3)}$. A role of dietary fat has been suggested in inflammation and oxidative stress $^{(4,5)}$. Because several features of the metabolic syndrome (MetS) are associated with enhanced inflammatory and oxidative stress markers ${ }^{(1,4,5)}$, it is relevant to investigate if these markers can be lowered by altering dietary fat intake.

Abbreviations: 15-keto-dihydro-PGF ${ }_{2 \alpha}, 15$-keto-13,14-dihydro-PGF $2 \alpha$; $\%$, \% energy; CRP, C-reactive protein; DPA, docosapentaenoic acid; HMUFA, high-fat diet rich in MUFA; HSFA, high-fat diet rich in SFA; LFHCC, low-fat high-complex carbohydrate diets; MetS, metabolic syndrome.

* Corresponding author: Dr U. Risérus, fax +461861179 76, email ulf.riserus@pubcare.uu.se 
Inflammatory processes can be investigated by measuring cytokine-mediated products such as C-reactive protein (CRP), or by studying the cyclo-oxygenase-induced pathway. Cyclo-oxygenase is an enzyme that catalyses the conversion of arachidonic acid to $\mathrm{PGF}_{2 \alpha}$, a potent smooth muscle stimulator and a vaso- and bronchoconstrictor. When secreted into the systemic circulation, $\mathrm{PGF}_{2 \alpha}$ is instantly converted to the major metabolite 15-keto-13,14-dihydro-PGF $\mathrm{PG}_{2 \alpha}$ (15-keto-dihydro$\mathrm{PGF}_{2 \alpha}$ ), and the latter is used to measure in vivo $\mathrm{PGF}_{2 \alpha}$ biosynthesis ${ }^{(4)}$. Isoprostanes are a group of PG-like compounds, but unlike PG, their formation is not cyclo-oxygenase dependent. 8-Iso-PGF ${ }_{2 \alpha}$ is the major $F_{2}$-isoprostane synthesised from arachidonic acid through a free radical-catalysed reaction, and is a reliable indicator of oxidative stress ${ }^{(5-7)}$.

The aim of the present study was to investigate the effects of an isoenergetic modification of dietary fat quantity and quality on concentrations of 8 -iso- $\mathrm{PGF}_{2 \alpha}$ (as an oxidative stress marker), 15-keto-dihydro- $\mathrm{PGF}_{2 \alpha}$ (as an indicator of cyclo-oxygenase-mediated inflammation) and CRP (as a marker of cytokine-induced inflammation) in subjects with the MetS.

\section{Methods}

\section{Study design}

The LIPGENE study is a randomised, parallel intervention study carried out at eight European centres: University College Dublin, Republic of Ireland; University of Reading, UK; Oslo University Hospital Aker/University of Oslo, Norway; INSERM, Marseille, France; Maastricht University, The Netherlands; Hospital Universitario Reina Sofía, University of Córdoba, Spain; Jagiellonian Medical University College, Krakow, Poland; and Uppsala University, Sweden. The study was conducted according to the guidelines laid down in the Declaration of Helsinki, registered with the US National Library of Medicine Clinical Trials registry (NCT00429195) and approved by the local ethics committees at each centre. All the participants gave written informed consent before entering the study.

A total of 486 volunteers with the MetS were randomised in this 12-week dietary intervention study. Inclusion criteria were age $35-70$ years, BMI $20-40 \mathrm{~kg} / \mathrm{m}^{2}$ and the MetS defined by three or more of the following criteria (National Cholesterol Education Program Adult Treatment Panel III ${ }^{(8)}$, slightly modified): fasting plasma glucose $>5.5 \mathrm{mmol} / \mathrm{l}$, serum $\mathrm{TAG} \geq 1.5 \mathrm{mmol} / \mathrm{l}$, serum $\mathrm{HDL}$-cholesterol $<1.0$ $\mathrm{mmol} / \mathrm{l}$ in males and $<1.3 \mathrm{mmol} / \mathrm{l}$ in females, waist circumference $>102 \mathrm{~cm}$ in males and $>88 \mathrm{~cm}$ in females, and elevated blood pressure (systolic blood pressure $\geq 130$ $\mathrm{mmHg}$, diastolic blood pressure $\geq 85 \mathrm{mmHg}$ or on prescribed blood pressure-lowering medication). Study design and recruitment strategies have been described previously ${ }^{(9)}$. Before and after intervention, all the subjects completed a health and lifestyle questionnaire, and anthropometric and biochemical measurements were performed according to standardised protocols.

The randomisation was performed centrally, according to age, sex and fasting plasma glucose concentration using the MINIM (Minimisation Programme for Allocating patients to Clinical Trials, Department of Clinical Epidemiology,
London Hospital Medical College, London, UK) randomisation programme. Participants were randomised to one of the four isoenergetic diets differing in fat quantity and quality: two high-fat diets $(38 \%$ energy $(\% \mathrm{E}))$ and two low-fat highcomplex carbohydrate diets $(28 \% \mathrm{E})$. Of the high-fat diets ( $38 \% \mathrm{E}$ fat), one was rich in saturated fat (HSFA: $16 \% \mathrm{E}$ SFA, $12 \% \mathrm{E}$ MUFA and $6 \% \mathrm{E}$ PUFA), and the other one was rich in monounsaturated fat (HMUFA: $8 \% \mathrm{E}$ SFA, $20 \% \mathrm{E}$ MUFA and $6 \% \mathrm{E}$ PUFA). The low-fat high-complex carbohydrate diets (LFHCC; $28 \% \mathrm{E}$ fat: $8 \% \mathrm{E} \mathrm{SFA,} 11 \% \mathrm{E}$ MUFA and $6 \% \mathrm{E}$ PUFA) were supplemented with either $1.24 \mathrm{~g} / \mathrm{d}$ very long chain $n-3$ PUFA (LFHCC $n-3$ ) (Marinol ${ }^{\circledR}$ $\mathrm{C}-38$, the EPA and DHA ratio was $1.4: 1$ ) or $1 \mathrm{~g} / \mathrm{d}$ high-oleic acid sunflower oil (LFHCC). To attain the targets of the dietary intervention, a food exchange model was used as described by Shaw et al. ${ }^{(9)}$. Before intervention, dietary habitual intake was assessed by a $3 \mathrm{~d}$ weighed food dietary record, and was used as a basis for isoenergetic $(<2 \mathrm{~kg}$ weight change) dietary fat modification. Trained nutritionists performed $24 \mathrm{~h}$ recalls every second week, and two additional $3 \mathrm{~d}$ weighed food dietary records were completed at weeks 6 and 12 to assess dietary compliance. Energy intake was adjusted if body weight change exceeded $2 \mathrm{~kg}$. Physical activity, alcohol consumption and smoking habits were not altered during the intervention.

Fasting urine samples were collected by the participants and brought during the visits. Anthropometric measurements were recorded according to a standardised protocol for the LIPGENE study. Blood pressure was measured using an automatic blood pressure device. Blood samples were taken after $12 \mathrm{~h}$ of fasting. Plasma TAG, HDL-cholesterol and glucose analyses were done with an ILAB 600 clinical chemical analyser using enzymatic colorometric kits (Instrumentation Laboratory, Warrington, UK). Serum CRP concentrations were determined by high-sensitivity ELISA (BioCheck, Inc., Foster City, CA, USA) at the University College Dublin. Urinary 15-keto-dihydro-PGF $2 \alpha{ }^{(10)}$ and 8-iso-PGF $2 \alpha{ }^{(11)}$ were assessed at the Uppsala University by RIA, and were adjusted for urinary creatinine (IL ${ }^{\mathrm{TM}}$ Test; Monarch International, Inc., Amherst, NH, USA). The intra-assay $\mathrm{CV}$ was $12.2 \%$ at low concentrations and $14 \cdot 0-14.5 \%$ at high concentrations.

\section{Statistics}

Baseline characteristics are described as means and standard deviations. Variables that are not normally distributed are described as median and interquartile range. 8-Iso-PGF $2 \alpha$, 15-keto-dihydro- $\mathrm{PGF}_{2 \alpha}$ and CRP were not normally distributed, so non-parametric statistics were used. Kruskal-Wallis test was performed to investigate differences in the inflammatory and oxidative stress markers between the diet groups. To test paired observations within each diet group, Wilcoxon signed-rank test was used. Subgroup analyses were performed according to sex and smoking habits. To avoid elevated CRP due to acute infections, subjects with CRP concentrations $\geq 10 \mathrm{mg} / \mathrm{l}$ at either baseline ( $n$ 60) or end of the study ( $n 49)$ were excluded from the post hoc analysis (HSFA, $n$ 81; HMUFA, $n$ 94; LFHCC, $n$ 84, and LFHCC $n-3, n$ 81). Two subanalyses were performed by dividing the participants according to the total fat intake above or below the median: one was based on fat intake at baseline, and the other was 
Table 1. Baseline characteristics of subjects who completed the intervention (Mean values and standard deviations)

\begin{tabular}{|c|c|c|c|c|c|c|c|c|}
\hline & \multicolumn{2}{|c|}{ HSFA ( $n$ 100) } & \multicolumn{2}{|c|}{ HMUFA ( $n$ 111) } & \multicolumn{2}{|c|}{ LFHCC $(n$ 106) } & \multicolumn{2}{|c|}{ LFHCC $n-3(n 100)$} \\
\hline & Mean & SD & Mean & SD & Mean & SD & Mean & SD \\
\hline Age (years) & 55 & 9 & 55 & 9 & 55 & 9 & 55 & 10 \\
\hline Female $(n)$ & \multicolumn{2}{|c|}{54} & \multicolumn{2}{|c|}{65} & \multicolumn{2}{|c|}{59} & \multicolumn{2}{|c|}{54} \\
\hline BMI $\left(\mathrm{kg} / \mathrm{m}^{2}\right)$ & 31.9 & 4.5 & $32 \cdot 4$ & $4 \cdot 3$ & 32.5 & $4 \cdot 2$ & $32 \cdot 4$ & $4 \cdot 2$ \\
\hline Waist circumference ${ }^{\star}(\mathrm{cm})$ & 105 & $98-113$ & 106 & $98-115$ & 105 & $99-114$ & 107 & $100-112$ \\
\hline Fasting glucose $(\mathrm{mmol} / \mathrm{l})$ & $5 \cdot 8$ & $5 \cdot 5-6 \cdot 4$ & $5 \cdot 7$ & $5 \cdot 4-6 \cdot 3$ & $5 \cdot 8$ & $5 \cdot 4-6 \cdot 3$ & $5 \cdot 8$ & $5 \cdot 5-6 \cdot 4$ \\
\hline $\mathrm{TAG}^{*}(\mathrm{mmol} / \mathrm{l})$ & 1.6 & $1 \cdot 2-2 \cdot 3$ & 1.6 & $1 \cdot 2-2 \cdot 2$ & 1.6 & $1 \cdot 1-2 \cdot 2$ & 1.6 & $1 \cdot 3-2 \cdot 0$ \\
\hline HDL-cholesterol* (mmol//) & $1 \cdot 0$ & $0.9-1 \cdot 2$ & 1.1 & $0.9-1.2$ & 1.1 & $0.9-1.3$ & $1 \cdot 1$ & $0.9-1 \cdot 3$ \\
\hline Systolic blood pressure $(\mathrm{mmHg})$ & $137 \cdot 8$ & $15 \cdot 3$ & $138 \cdot 9$ & $16 \cdot 2$ & 138.4 & $15 \cdot 4$ & $137 \cdot 6$ & 14.5 \\
\hline Diastolic blood pressure $(\mathrm{mmHg})$ & $86 \cdot 2$ & $8 \cdot 8$ & $86 \cdot 0$ & 9.5 & 84.9 & $9 \cdot 2$ & 85.5 & 8.5 \\
\hline 8-Iso-PGF ${ }_{2 \alpha}{ }^{*}(\mathrm{nmol} / \mathrm{mmol}$ creatinine $)$ & 0.43 & $0.36-0.54$ & 0.46 & $0.38-0.57$ & 0.44 & $0.37-0.53$ & 0.45 & $0.38-0.54$ \\
\hline $\begin{array}{l}\text { 15-Keto-dihydro-PGF }{ }_{2 \alpha}{ }^{*} \\
\text { (nmol/mmol creatinine) }\end{array}$ & $0 \cdot 16$ & $0.12-0.19$ & 0.17 & $0 \cdot 14-0.21$ & 0.15 & $0.12-0.18$ & 0.15 & $0 \cdot 12-0 \cdot 20$ \\
\hline C-reactive protein* (mg/l) & $3 \cdot 6$ & $2 \cdot 3-7 \cdot 3$ & 3.7 & $1 \cdot 8-7 \cdot 0$ & 3.9 & $2 \cdot 1-7 \cdot 6$ & 4.4 & $2 \cdot 0-6 \cdot 7$ \\
\hline
\end{tabular}

HSFA, high-fat diet rich in SFA; HMUFA, high-fat diet rich in MUFA; LFHCC, low-fat high-complex carbohydrate diet; LFHCC $n$-3, LFHCC with $n$-3 supplementation.

* Variables that are not normally distributed are presented as medians and interquartile ranges.

based on fat intake in each diet group at follow-up. Kruskal-Wallis test and Wilcoxon signed-rank test were also performed to investigate differences in plasma fatty acid composition as a measurement of compliance. The change in major plasma fatty acids (i.e. $\%_{\text {(at follow-up) }}-\%_{\text {(at baseline) }}$ ) was associated with changes in oxidative stress and inflammatory markers (i.e. concentration $_{(\text {at follow-up) }}-$ concentration $\left._{(\text {at baseline })}\right)$ by Spearman's correlation. The statistical analyses were carried out using the software SAS, version 10 (SAS Institute, Inc., NC, USA). All tests were two tailed, and $P<0.05$ was considered statistically significant.

\section{Results}

A total of 417 subjects completed the study. Measures of CRP, 15-keto-dihydro-PGF ${ }_{2 \alpha}$ and 8 -iso- $\mathrm{PGF}_{2 \alpha}$ concentrations at both baseline and follow-up were assessed in 415, 408 and 409 subjects, respectively. Baseline characteristics of the subjects fulfilling the intervention are presented in Table 1. Seventy-three subjects reported the use of tobacco (HSFA, n 22 (22\%); HMUFA, $n 22$ (20\%); LFHCC, $n 15$ (14\%); and LFHCC $n-3, n 14(14 \%))$. The median for the number of cigarettes consumed per day was 11 (minimum: $0 \cdot 1$ and maximum: 60). Dietary intake at the end of the study is presented in Table 2. Fatty acid composition in the plasma at the end of the study is presented in Table 3. At the end of the study, the HMUFA group had higher proportions of oleic acid $(18: 1)$ in the plasma than the HSFA and LFHCC $n-3$ groups $(P=0.0008$ and $0 \cdot 005$, respectively). There was also a trend towards a higher proportion of $18: 1$ in the HMUFA group than in the LFHCC group $(P=0 \cdot 06)$. With regard to the $n-3$ PUFA, the LFHCC $n-3$ group had significantly higher proportions of EPA $(P=0.0001)$, docosapentaenoic acid (DPA) $(P<0.05)$ and DHA $(P<0.003)$ than the other groups, except for DHA, which did not differ significantly between the two low-fat diets. Neither concentrations of 8-iso-PGF $2 \alpha$ and 15-keto-dihydro- $\mathrm{PGF}_{2 \alpha}$ nor those of CRP differed between the diet groups at baseline $(P>0.07)$ or at the end of the study $(P>0.18)$. The investigated inflammatory and oxidative stress markers were not altered during the intervention (Table 4), and no differences in changes were observed between the diet groups (8-iso-PGF $\mathrm{P}_{2 \alpha}$, $P=0.83$; 15 -keto-dihydro-PGF ${ }_{2 \alpha}, P=0.45$; and CRP, $P=0.97$ (results on CRP have been reported previously briefly as 'data not shown' AC Tierney, unpublished results)). Results were not altered in the post hoc analyses based on smoking status or sex. Furthermore, no significant differences were observed

Table 2. Dietary intake at follow-up for subjects who completed the intervention

(Aims, medians and interquartile ranges (IQR))

\begin{tabular}{|c|c|c|c|c|c|c|c|c|c|c|c|c|}
\hline & \multicolumn{3}{|c|}{ HSFA ( $n$ 97) } & \multicolumn{3}{|c|}{ HMUFA $(n 110)$} & \multicolumn{3}{|c|}{ LFHCC $(n 104)$} & \multicolumn{3}{|c|}{ LFHCC $n-3(n 99)$} \\
\hline & Aim & Median & IQR & Aim & Median & IQR & Aim & Median & IQR & Aim & Median & IQR \\
\hline Energy (MJ) & & 8.5 & $7 \cdot 1-10 \cdot 1$ & & $8 \cdot 2$ & $6 \cdot 3-10.5$ & & $8 \cdot 1$ & $6.5-9.4$ & & $8 \cdot 2$ & $6 \cdot 5-10 \cdot 0$ \\
\hline Fat $(\% \mathrm{E})$ & 38 & 39.8 & $37 \cdot 1-42 \cdot 2$ & 38 & 39.5 & $36 \cdot 0-42 \cdot 0$ & 28 & $29 \cdot 2$ & $25 \cdot 9-33 \cdot 3$ & 28 & $28 \cdot 2$ & $25 \cdot 8-30 \cdot 7$ \\
\hline SFA (\%E) & 16 & $17 \cdot 6$ & $15 \cdot 6-18 \cdot 8$ & 8 & $10 \cdot 2$ & $8 \cdot 9-11 \cdot 8$ & 8 & 8.5 & $6 \cdot 7-10 \cdot 4$ & 8 & 8.4 & $6 \cdot 8-10 \cdot 4$ \\
\hline MUFA (\%E) & 12 & $12 \cdot 5$ & $11 \cdot 1-14 \cdot 0$ & 20 & $19 \cdot 1$ & $15 \cdot 7-21 \cdot 7$ & 11 & $11 \cdot 8$ & $10 \cdot 1-13 \cdot 2$ & 11 & $11 \cdot 1$ & $9 \cdot 7-12 \cdot 3$ \\
\hline PUFA (\%E) & 6 & $6 \cdot 4$ & $5 \cdot 0-7 \cdot 2$ & 6 & $6 \cdot 3$ & $5 \cdot 5-7.5$ & 6 & $5 \cdot 9$ & $4 \cdot 8-7 \cdot 2$ & 6 & 5.5 & $4 \cdot 7-6 \cdot 5$ \\
\hline Carbohydrate (\%E) & & 41.9 & $36 \cdot 8-45 \cdot 9$ & & $42 \cdot 0$ & $38 \cdot 0-46 \cdot 0$ & & $49 \cdot 1$ & $45 \cdot 7-54 \cdot 3$ & & $51 \cdot 6$ & $45 \cdot 8-54 \cdot 6$ \\
\hline Protein (\%E) & & $17 \cdot 1$ & $14 \cdot 4-20 \cdot 2$ & & $16 \cdot 6$ & $15 \cdot 4-18 \cdot 6$ & & $18 \cdot 0$ & $15 \cdot 7-21 \cdot 3$ & & $17 \cdot 9$ & $15 \cdot 5-20 \cdot 3$ \\
\hline Alcohol (\%E) & & 0.0 & $0.0-2.5$ & & 0.0 & $0.0-3.2$ & & 0.0 & $0.0-2 \cdot 0$ & & 0.3 & $0.0-2 \cdot 3$ \\
\hline
\end{tabular}

HSFA, high-fat diet rich in SFA; HMUFA, high-fat diet rich in MUFA; LFHCC, low-fat high-complex carbohydrate diet; LFHCC $n$-3, LFHCC with $n$-3 supplementation; $\% \mathrm{E}$, percentage energy. 
Table 3. Fatty acid composition in the plasma at the end of the intervention (Medians and interquartile ranges (IQR))

\begin{tabular}{|c|c|c|c|c|c|c|c|c|}
\hline \multirow[b]{2}{*}{ Plasma fatty acid (\%) } & \multicolumn{2}{|c|}{ HSFA } & \multicolumn{2}{|c|}{ HMUFA } & \multicolumn{2}{|c|}{ LFHCC } & \multicolumn{2}{|c|}{ LFHCC $n-3$} \\
\hline & Median & IQR & Median & IQR & Median & IQR & Median & IQR \\
\hline $14: 0$ & 2.5 & $1.6-3.5$ & $2 \cdot 1$ & $1 \cdot 3-3 \cdot 3$ & $2 \cdot 1$ & $1.4-3.3$ & $2 \cdot 3$ & $1.6-3.5$ \\
\hline $16: 0$ & $24 \cdot 7$ & $21 \cdot 8-28 \cdot 2$ & $24 \cdot 0$ & $22 \cdot 1-26 \cdot 9$ & $24 \cdot 8$ & $22 \cdot 3-27 \cdot 7$ & 24.5 & $21 \cdot 5-27 \cdot 1$ \\
\hline $16: 1$ & 1.0 & $0.6-1.6$ & 1.0 & $0.6-1.8$ & $1 \cdot 2$ & $0.7-1.6$ & $1 \cdot 1$ & $0.6-1.6$ \\
\hline $18: 0$ & 3.9 & $2 \cdot 3-4 \cdot 8$ & 3.6 & $2 \cdot 2-4 \cdot 8$ & 3.5 & $2 \cdot 2-4 \cdot 6$ & 3.5 & $2 \cdot 1-4 \cdot 7$ \\
\hline $18: 1$ & $24 \cdot 8$ & $22 \cdot 4-28 \cdot 0$ & $27 \cdot 2$ & $24 \cdot 5-30 \cdot 0$ & $26 \cdot 4$ & $23 \cdot 0-29 \cdot 2$ & $25 \cdot 6$ & $22 \cdot 6-28 \cdot 2$ \\
\hline $18: 2 n-6$ & $28 \cdot 8$ & $25 \cdot 7-32 \cdot 2$ & $27 \cdot 1$ & $24.1-30.9$ & $28 \cdot 2$ & $24 \cdot 3-31 \cdot 4$ & $27 \cdot 3$ & $24.4-30.9$ \\
\hline $18: 3 n-6$ & 0.02 & $0.01-0.05$ & 0.03 & $0.01-0.07$ & 0.03 & $0.01-0.07$ & 0.03 & $0.02-0.06$ \\
\hline $18: 3 n-3$ & 0.03 & $0.02-0.09$ & 0.02 & $0.01-0.06$ & 0.03 & $0.01-0.06$ & 0.04 & $0.01-0.07$ \\
\hline $20: 3 n-6$ & 1.0 & $0.5-1.5$ & 0.9 & $0.4-1.5$ & 0.8 & $0.4-1.3$ & 0.8 & $0.4-1.2$ \\
\hline $20: 4 n-6$ & $7 \cdot 1$ & $6 \cdot 3-8 \cdot 2$ & 7.5 & $5 \cdot 9-8 \cdot 8$ & $7 \cdot 3$ & $6 \cdot 3-8 \cdot 3$ & $6 \cdot 8$ & $5 \cdot 7-7 \cdot 8$ \\
\hline $20: 5 n-3$ & 0.8 & $0.5-1.1$ & 0.8 & $0.5-1.2$ & 0.9 & $0.5-1.6$ & 1.9 & $1 \cdot 2-2 \cdot 7$ \\
\hline $22: 5 n-3$ & 0.5 & $0.3-0.7$ & 0.5 & $0.4-0.6$ & 0.5 & $0.4-0.7$ & 0.6 & $0.5-0.8$ \\
\hline $22: 6 n-3$ & $2 \cdot 3$ & $1 \cdot 3-3 \cdot 0$ & $2 \cdot 3$ & $1.4-3.3$ & 2.5 & $1 \cdot 6-3 \cdot 8$ & $3 \cdot 2$ & $2 \cdot 0-4 \cdot 1$ \\
\hline
\end{tabular}

HSFA, high-fat diet rich in SFA; HMUFA, high-fat diet rich in MUFA; LFHCC, low-fat high-complex carbohydrate diet; LFHCC $n$-3, LFHCC with $n$-3 supplementation.

after classifying the subjects according to their habitual fat intake. Participants were also divided according to the total fat intake above or below the median in each diet group, respectively (medians: HSFA, 39.8\% E; HMUFA, 39.5\%E; LFHCC, $29.2 \% \mathrm{E}$; and LFHCC $n-3,28 \cdot 2 \% \mathrm{E}$ ). Subjects with a fat intake above the median in the LFHCC $n-3$ group had increased concentrations of 8 -iso- $\mathrm{PGF}_{2 \alpha}(P=0.05)$ during the intervention, whereas those with a fat intake above the median in the HMUFA group had low concentrations of 15-keto-dihydro$\mathrm{PGF}_{2 \alpha}(P=0.02)$.

The change in concentrations of 15-keto-dihydro-PGF $2 \alpha$ and CRP during the intervention was not related to changes in plasma fatty acids, whereas the change in 8 -iso- $\mathrm{PGF}_{2 \alpha}$ was negatively related to changes in DPA and DHA $(r-0 \cdot 15$, $P=0.003$ and $r-0.13, P=0.007$, respectively). When dividing the cohort into the diet groups, the associations remained for the two low-fat groups (LFHCC: $r-0 \cdot 27, P=0.005$ (DPA) and $r-0.21, P=0.03$ (DHA); LFHCC $n-3: r-0.27, P=0.01$ (DPA) and $r-0.25, P=0.02$ (DHA)), whereas the associations were non-significant $(P>0 \cdot 13)$ in the two high-fat groups.

\section{Discussion}

LIPGENE is a European multicentre dietary intervention study that investigates the effects of fat quality and quantity on risk factors associated with the MetS. Here, we present the results concerning the markers of oxidative stress and inflammation. The current dietary fat intervention, comparing high-fat diets (MUFA or SFA) $v$. low-fat diets (with or without $n-3$ supplementation), did not significantly alter the concentrations of 8-iso-PGF ${ }_{2 \alpha}$, 15-keto-dihydro-PGF ${ }_{2 \alpha}$ and CRP. However, the levels of 8 -iso- $\mathrm{PGF}_{2 \alpha}$ increased in subjects in the LFHCC $n-3$ group with a fat intake above the median $(28.2 \% \mathrm{E})$, but this should be interpreted with caution since we did not observe any similar trend elsewhere (e.g. when dividing this diet group according to their habitual fat intake). This is also valid for the observed decrease in 15-keto-dihydro-PGF $2 \alpha$ levels in the HMUFA group with a high fat intake.

An impact of fat quality on oxidative stress and inflammation has been investigated in other studies. After substituting a diet high in SFA with a diet high in linoleic acid $(18: 2 n-6,11.5 \% \mathrm{E}), 8$-iso- $\mathrm{PGF}_{2 \alpha}$ concentrations increased, whereas they remained unchanged after substitution with $\mathrm{MUFA}^{(12)}$. A possible explanation for this increase in oxidative stress may be that linoleic acid is a precursor of arachidonic acid (20:4n-6, the major substrate for isoprostane formation). Also, PUFA has been suggested to be more prone to oxidation than SFA and MUFA ${ }^{(13)}$. Excretion and formation of 8 -iso- $\mathrm{PGF}_{2 \alpha}$ were, on the other hand, not

Table 4. Within-group changes of the inflammatory and oxidative stress markers during the intervention, i.e. (concentration at follow-up) - (concentration at baseline)

(Medians and interquartile ranges (IQR))

\begin{tabular}{|c|c|c|c|c|c|c|c|c|c|c|c|c|}
\hline & \multicolumn{3}{|c|}{ HSFA } & \multicolumn{3}{|c|}{ HMUFA } & \multicolumn{3}{|c|}{ LFHCC } & \multicolumn{3}{|c|}{ LFHCC $n-3$} \\
\hline & $n$ & Median & IQR & $n$ & Median & IQR & $n$ & Median & IQR & $n$ & Median & IQR \\
\hline 8-Iso-PGF $2{ }_{2 \alpha}{ }^{*}$ & 95 & 0.01 & $-0.10-0.09$ & 110 & 0.00 & $-0.09-0.11$ & 106 & 0.00 & $-0.06-0.10$ & 98 & 0.01 & $-0.06-0.13$ \\
\hline 15-Keto-dihydro-PGF ${ }_{2 \alpha}$ * & 95 & 0.00 & $-0.02-0.03$ & 110 & 0.00 & $-0.04-0.02$ & 105 & 0.00 & $-0.03-0.02$ & 98 & 0.00 & $-0.03-0.02$ \\
\hline CRP†‡ & 99 & -0.1 & $-1 \cdot 2-1 \cdot 2$ & 110 & 0.1 & $-1 \cdot 2-1 \cdot 3$ & 106 & 0.0 & $-1.5-1.3$ & 100 & -0.1 & $-1.4-1.3$ \\
\hline
\end{tabular}

HSFA, high-fat diet rich in SFA; HMUFA, high-fat diet rich in MUFA; LFHCC, low-fat high-complex carbohydrate diet; LFHCC $n$-3, LFHCC with $n$-3 supplementation; CRP, C-reactive protein.

${ }^{*}$ Measured in urine $(\mathrm{nmol} / \mathrm{mmol}$ creatinine).

$\dagger$ Measured in plasma ( $\mathrm{mg} / \mathrm{l})$.

$\ddagger$ CRP has previously been presented as 'data not shown' (AC Tierney, unpublished results). 
changed after ingestion of SFA diet compared with a rapeseed oil diet rich in $\alpha$-linolenic acid $(18: 3 n-3)^{(14)}$. Contrary to the present results, daily supplementation of EPA and DHA for 3 months reduced the levels of 8 -iso- $\mathrm{PGF}_{2 \alpha}$ measured in the plasma, but not the levels of 15-keto-dihydro-PGF $2 \alpha$ compared with the controls among the healthy subjects in the KANWU study ${ }^{(15)}$. An isoprostane-reducing effect of $n-3$ PUFA has also been observed both in treated hypertensive subjects with type 2 diabetes $^{(16)}$ and in other subjects ${ }^{(5)}$. Our observed correlations between the change in 8-iso-PGF $2 \alpha$ and the changes in plasma DPA $(22: 5 n-3)$ and DHA $(22: 6 n-3)$ are in line with the previous results. Although the duration of supplementation was similar to that followed in the KANWU study, the dose of $n-3$ PUFA supplementation used in the present study might be too low to elicit an effect on 8 -iso-PGF $\mathrm{PG}_{2 \alpha}$ and 15-keto-dihydro- $\mathrm{PGF}_{2 \alpha}$ status. For example, the dose was less than half of that used in the KANWU study ${ }^{(15)}$. The impact of supplementation dose has also been discussed elsewhere ${ }^{(17)}$. Another explanation for the lack of effect may be that the endogenous levels of $n-3$ PUFA were sufficient already at baseline, while the dietinduced changes in circulating $n-3$ PUFA levels were limited, i.e. inadequate supplementation with respect to the preexisting intake ( $n-3$ PUFA at baseline was $3.6 \%$ (median), interquartile range: $2 \cdot 5-4.9 \%$ ).

The LIPGENE study is one of the largest dietary interventions in subjects with the MetS. Although the primary endpoint was insulin sensitivity, our post-trial power calculations did not indicate a lack of power to detect significant differences in inflammatory or oxidative stress markers. According to dietary records, compliance was high in all the diet groups ${ }^{(9)}$. However, fat intake assessment by dietary records is associated with reporting bias and measurement errors ${ }^{(18)}$, therefore compliance level should always be considered in all the dietary interventions. It should also be noted that the LIPGENE study is a multicentre study of a Pan-European nature, where there were differences between the habitual diets at baseline. Therefore, there may have been heterogeneity in the change of the dietary composition within each diet group, which may have affected the results. However, the lack of effect of dietary fat modification in this large randomised intervention trial is of clear scientific interest. To further elucidate the effect of various dietary fatty acids on oxidative stress and inflammatory markers, more specifically designed trials are needed, using other types of fatty acids, e.g. PUFA ( $n-6$ and/or $n-3$ ) instead of MUFA as well as applying higher doses of $n$-3 PUFA supplementation. It should also be mentioned that even though no effects were observed on the investigated systemic markers for oxidative stress and inflammation, there may be local tissue effects on cellular levels.

In conclusion, this 12-week dietary intervention did not show any effect of modified fat quantity and quality on the investigated oxidative stress and inflammatory markers $\left(\mathrm{F}_{2}\right.$-isoprostane, $\mathrm{PGF}_{2 \alpha}$ metabolite and $\left.\mathrm{CRP}\right)$ among subjects with the MetS.

\section{Acknowledgements}

We would like to thank all the persons involved in the LIPGENE project at all the clinical centres. J. M., H. L. G., A. C. T., S. M., O. H., D. I. S., J. A. R., J. L.-M., B. K.-W.,
I. G., E. E. B., W. H. M. S., C. A. D., J. A. L. and H. M. R. designed and organised the original LIPGENE study and data collection. U. R. and H. P. conceived the present substudy and drafted the manuscript. H. P. performed the statistical analyses, and S. B. analysed the oxidative stress markers. All the authors contributed to critical revision of the manuscript. The work was supported by LIPGENE - an European Union 6th Framework Program Integrated Project (FOOD-CT-2003-505944), NordForsk (Nordic Centre of Excellence in Food, Nutrition and Health (SYSDIET)), Swedish Nutrition Foundation and the Norwegian Foundation for Health and Rehabilitation. None of the authors has any conflicts of interest.

\section{References}

1. Pickup JC (2004) Inflammation and activated innate immunity in the pathogenesis of type 2 diabetes. Diabetes Care 27, 813-823.

2. Helmersson J, Vessby B, Larsson A, et al. (2004) Association of type 2 diabetes with cyclooxygenase-mediated inflammation and oxidative stress in an elderly population. Circulation $\mathbf{1 0 9}$, 1729-1734.

3. Jialal I, Devaraj S \& Venugopal SK (2002) Oxidative stress, inflammation, and diabetic vasculopathies: the role of alpha tocopherol therapy. Free Radic Res 36, 1331-1336.

4. Basu S (2007) Novel cyclooxygenase-catalyzed bioactive prostaglandin F2alpha from physiology to new principles in inflammation. Med Res Rev 27, 435-468.

5. Basu S (2008) F2-isoprostanes in human health and diseases: from molecular mechanisms to clinical implications. Antioxid Redox Signal 10, 1405-1434.

6. Kadiiska MB, Gladen BC, Baird DD, et al. (2005) Biomarkers of oxidative stress study II: are oxidation products of lipids, proteins, and DNA markers of $\mathrm{CCl}_{4}$ poisoning? Free Radic Biol Med 38, 698-710.

7. Kadiiska MB, Gladen BC, Baird DD, et al. (2005) Biomarkers of oxidative stress study III. Effects of the nonsteroidal antiinflammatory agents indomethacin and meclofenamic acid on measurements of oxidative products of lipids in $\mathrm{CCl}_{4}$ poisoning. Free Radic Biol Med 38, 711-718.

8. Grundy SM, Brewer HB Jr, Cleeman JI, et al. (2004) Definition of metabolic syndrome: report of the National Heart, Lung, and Blood Institute/American Heart Association conference on scientific issues related to definition. Arterioscler Thromb Vasc Biol 24, e13-e18.

9. Shaw DI, Tierney AC, McCarthy S, et al. (2009) LIPGENE food-exchange model for alteration of dietary fat quantity and quality in free-living participants from eight European countries. Br J Nutr 101, 750-759.

10. Basu S (1998) Radioimmunoassay of 15-keto-13,14-dihydroprostaglandin F2alpha: an index for inflammation via cyclooxygenase catalysed lipid peroxidation. Prostaglandins Leukot Essent Fatty Acids 58, 347-352.

11. Basu S (1998) Radioimmunoassay of 8-iso-prostaglandin F2alpha: an index for oxidative injury via free radical catalysed lipid peroxidation. Prostaglandins Leukot Essent Fatty Acids 58, 319-325.

12. Turpeinen AM, Basu S \& Mutanen M (1998) A high linoleic acid diet increases oxidative stress in vivo and affects nitric oxide metabolism in humans. Prostaglandins Leukot Essent Fatty Acids 59, 229-233.

13. Wagner BA, Buettner GR \& Burns CP (1994) Free radicalmediated lipid peroxidation in cells: oxidizability is a function of cell lipid bis-allylic hydrogen content. Biochemistry $\mathbf{3 3}$ $4449-4453$. 
14. Sodergren E, Gustafsson IB, Basu S, et al. (2001) A diet containing rapeseed oil-based fats does not increase lipid peroxidation in humans when compared to a diet rich in saturated fatty acids. Eur J Clin Nutr 55, 922-931.

15. Nalsen C, Vessby B, Berglund L, et al. (2006) Dietary (n-3) fatty acids reduce plasma F2-isoprostanes but not prostaglandin F2alpha in healthy humans. J Nutr 136, 1222-1228.

16. Mori TA, Woodman RJ, Burke V, et al. (2003) Effect of eicosapentaenoic acid and docosahexaenoic acid on oxidative stress and inflammatory markers in treated-hypertensive type 2 diabetic subjects. Free Radic Biol Med 35, 772-781.

17. Galli C \& Calder PC (2009) Effects of fat and fatty acid intake on inflammatory and immune responses: a critical review. Ann Nutr Metab 55, 123-139.

18. Hodson L, Skeaff CM \& Fielding BA (2008) Fatty acid composition of adipose tissue and blood in humans and its use as a biomarker of dietary intake. Prog Lipid Res 47, $348-380$. 